\title{
First CDF II Heavy Flavor physics results with the Silicon Vertex Trigger
}

\author{
A. Cerri $^{\mathrm{a} *}$
}

a Physics Department, Lawrence Berkeley National Laboratory, 1 Cyclotron road, 94720 Berkeley, CA, USA

The renewed CDF II experiment recently re-started data taking. The data collected lately already provides insight in the heavy flavor capabilities of this $p \bar{p}$ experiment.

As a benchmark of these possibilities we present two preliminary results: the measurement of the branching fractions $B R\left(D^{\circ} \rightarrow \pi^{+} \pi^{-}\right)$and $B R\left(D^{\circ} \rightarrow K^{+} K^{-}\right)$relative to $B R\left(D^{\circ} \rightarrow K^{ \pm} \pi^{\mp}\right)$ and the measurement of the invariant mass difference between $D_{s}^{ \pm} \rightarrow \phi \pi^{ \pm}, \phi \rightarrow K^{+} K^{-}$and $D^{ \pm} \rightarrow \phi \pi^{ \pm}, \phi \rightarrow K^{+} K^{-}$. The preliminary results are $\frac{B R\left(D^{\circ} \rightarrow \pi^{+} \pi^{-}\right)}{B R\left(D^{\circ} \rightarrow K^{ \pm} \pi^{\mp}\right)}=3.37 \pm 0.20($ stat $) \pm 0.16$ (sys) \%, $\frac{B R\left(D^{\circ} \rightarrow K^{+} K^{-}\right)}{B R\left(D^{\circ} \rightarrow K^{ \pm} \pi^{\mp}\right)}=11.17 \pm 0.48($ stat $) \pm 0.98($ sys $) \%$ and $\Delta m\left(D_{s}-D^{+}\right)=99.28 \pm 0.43($ stat $) \pm 0.27$ (sys) $\mathrm{MeV} / \mathrm{c}^{2}$. All results are consistent with PDG expectations.

\section{Introduction}

A detailed description of the renewal of the CDF experiment commonly referred to as "CDF II" can be found elsewhere [1]. In this paper we will focus mainly on the physics results that the experiment has obtained so far exploiting the new Silicon Vertex Trigger based selections.

The Silicon Vertex Trigger is one of the major upgrades of CDF II: it consists of a real-time tracking device capable of reconstructing $2 \mathrm{D}$ silicon tracks with offline-quality resolution [1].

This allows the selection of low momentum heavy flavor enriched events: lifetime based selections like impact parameter cuts are in fact a well known tool for discriminating $b \bar{b}$ and $c \bar{c}$ physics events from the mostly prompt hadronic background typical of hadron colliders $[2,3]$.

The results described here have to be considered preliminary benchmarks rather than full-fledged analyses: at the moment of writing this proceeding we already know that statistical and systematical errors will be better under control in the future. Nevertheless this is the first time that non-leptonic heavy flavor physics is collected efficiently at an hadron collider: this will be the key to competitivity in the beauty and charm sectors for CDF II.

\section{Charm physics}

The charm production cross section at the momentum range explorable with the hadronic $B$ trigger (SVT trigger from now on) is as large as uncertain [4]. At this stage we know that the amount of promptly produced charm is quite sizeable (see figure 1). Also Cabibbo suppressed de-

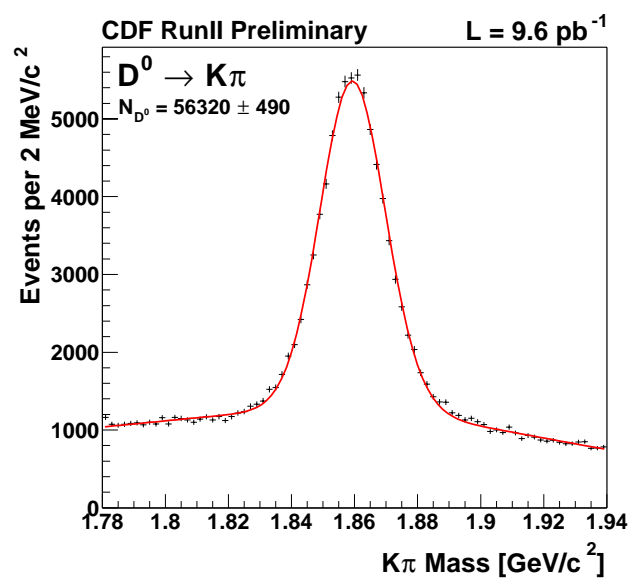

Figure 1. Offline reconstructed invariant mass distribution of the two trigger tracks. Note the huge collection rate of $D^{\circ}$ decays: this is so large that we can identify candidates in data samples collected in few hours.

\footnotetext{
${ }^{*}$ on behalf of the CDFII collaboration
} 
cays to CP eigenstates (like $K K$ and $\pi \pi$ ) are collected at such huge rates with exactly the same trigger: this suggests the possibility of directly comparing branching fractions into the three possible different 2-body final states containing two charged tracks: $D^{\circ} \rightarrow K^{ \pm} \pi^{\mp}, D^{\circ} \rightarrow K^{+} K^{-}$, $D^{\circ} \rightarrow \pi^{+} \pi^{-}$. Systematics are in fact minimized requiring a common set of selection and reconstruction cuts, while intrinsic differences coming from the slight difference in the physics of the final states can be taken into account properly through detector simulation, and then treated as an additional systematics uncertainty.

The interest in this measurement is three prong: (i) first of all it is a thermometer of the CDF II performances (ii) secondly, it is the first step towards interesting charm physics measurements such as the experimental determination of the $c \bar{c}$ parameter $y$ [5] and last but not least, (iii) it provides a very important control sample for the study of the detector performances (particle id in particular) for the reconstruction of two prong

\begin{tabular}{lcc}
\hline Systematic source & $\frac{D^{\circ} \rightarrow K^{+} K^{-}}{D^{\circ} \rightarrow K^{ \pm} \pi^{\mp}}$ & $\frac{D^{\circ} \rightarrow \pi^{+} \pi^{-}}{D^{\circ} \rightarrow K^{ \pm} \pi^{\mp}}$ \\
\hline Background model & $7.9 \%$ & $1.4 \%$ \\
Signal model & $1.3 \%$ & $1.3 \%$ \\
\hline Trigger simulation & $1.9 \%$ & $2.5 \%$ \\
Beam $z$ profile & $0.7 \%$ & $0.5 \%$ \\
Material description & $0.5 \%$ & $0.5 \%$ \\
\hline Lifetime difference & $2.2 \%$ & $2.2 \%$ \\
DCS Decays & $0.4 \%$ & $0.4 \%$ \\
\hline Input $D^{\circ}$ spectra & $1.8 \%$ & $1.8 \%$ \\
$b / c$ ratio & $1.1 \%$ & - \\
Tracking & $0.4 \%$ & $1.7 \%$ \\
\hline Total & $8.8 \%$ & $4.6 \%$ \\
\hline
\end{tabular}

Table 1

Systematic uncertainty contribution for the measurement of the relative BR of $D^{\circ} \rightarrow K^{+} K^{-}$and $D^{\circ} \rightarrow \pi^{+} \pi^{-}$WRT $D^{\circ} \rightarrow K^{ \pm} \pi^{\mp}$

charmless $B$ decays.

The relative branching fractions are measured fitting the invariant mass distributions of the candidates with suitable signal and background models. The measurement of the relative selection and reconstruction efficiency relies on detector and trigger simulation, with the uncertainty on the input spectra and on the bottom to charm relative contribution being treated as additional systematics.

In the final systematics evaluation also the effect of possible differences in the states lifetimes and the contribution from DCS decays are accounted. Even with the limited integrated luminosity used in this study $\left(\approx 10.5 p b^{-1}\right)$, CDF II is able to measure the relative branching fractions with an accuracy comparable to the best $c \bar{c}$ results available in literature [5]: for instance the CLEO II results [6] obtained with $10 \mathrm{fb}^{-1}$ of integrated luminosity at the $\Upsilon(4 s)$ resonance. The preliminary results are:

$$
\begin{aligned}
& \frac{B R\left(D^{\circ} \rightarrow \pi^{+} \pi^{-}\right)}{B R\left(D^{\circ} \rightarrow K^{ \pm} \pi^{\mp}\right)}=3.37 \pm 0.20(\text { stat }) \pm 0.16(\text { sys }) \% \\
& \frac{B R\left(D^{\circ} \rightarrow K^{+} K^{-}\right)}{B R\left(D^{\circ} \rightarrow K^{ \pm} \pi^{\mp}\right)}=11.17 \pm 0.48(\text { stat }) \pm 0.98(\text { sys }) \%
\end{aligned}
$$

Systematic uncertainty in our measurement is to date dominated by the background modeling systematics in the resonance fit for $D^{\circ} \rightarrow$ $K^{+} K^{-}$and by the trigger simulation systematics in $D^{\circ} \rightarrow \pi^{+} \pi^{-}$. The detailed breakdown of the systematics uncertainties is reported in table 1 .

\section{Charm and Charm-Strange signals}

Analogously to what happens for the different decay modes of the $D^{\circ}$, also the systematics in the

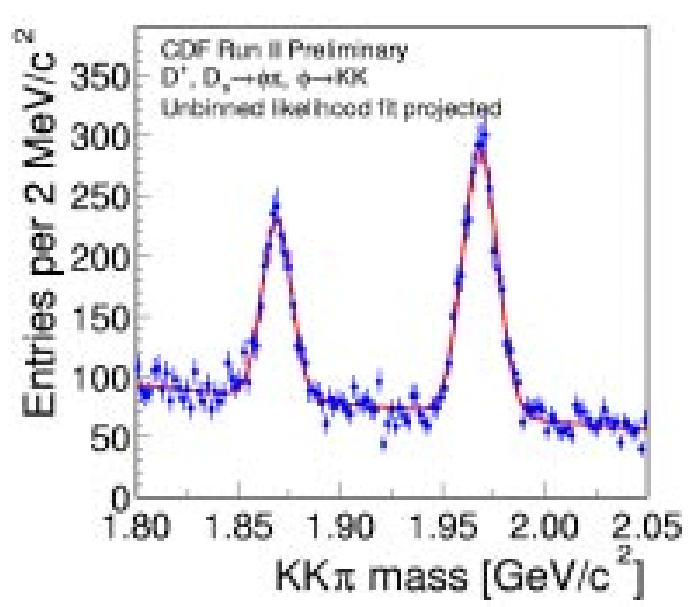

Figure 2. Offline reconstructed invariant mass distribution of the $\phi \pi$ candidates. 


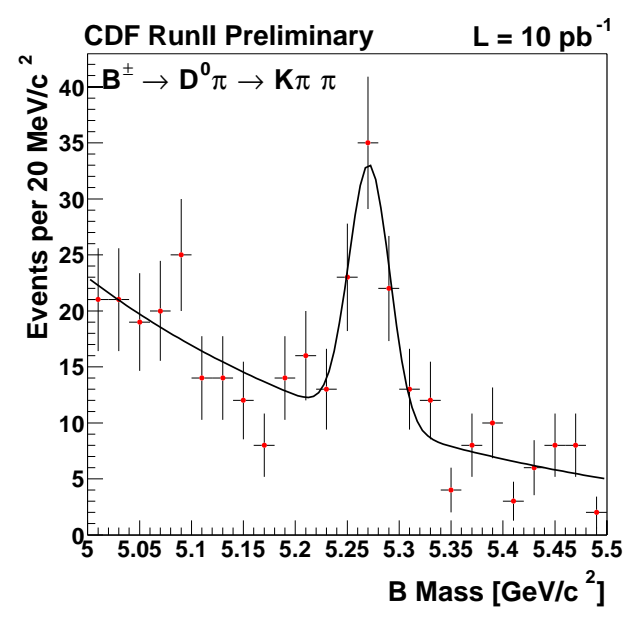

Figure 3. Invariant mass distribution for the $B^{ \pm} \rightarrow D^{\circ} \pi^{ \pm}$decay, after appropriate selections.

extraction of the $D_{s}-D^{+}$mass difference can be reduced selecting a configuration where the decay mode and the trigger selection are identical. This is the case of $D^{ \pm}, D_{s}^{ \pm} \rightarrow \phi \pi^{ \pm}, \phi \rightarrow K^{+} K^{-} . D^{+}$ and $D_{s}$ are fitted in $11.6 \mathrm{pb}^{-1}$ of data, after correcting for charged tracks energy loss. This measurement gives a feeling of our ability in achieving precise mass measurements. The preliminary result is:

$\Delta m=99.28 \pm 0.43$ (stat) \pm 0.27 (sys) $\mathrm{MeV} / \mathrm{c}^{2}$

The most important contributions to systematics are coming from selection/reconstruction cuts ( $\chi^{2}$ for the $\phi \pi$ combined fit, transverse plane decay length, and helicity angle are the three main variables which could introduce biases related to the small kinematic differences between $D^{+}$and $D_{s}$ ) and from the modeling of the resonances used to extract the mass differences. Tracking systematics are also important (uncertainty on the scale of the track parameters covariance matrix, detector material uncertainty, the magnetic field scale and tracking biases related to alignment all contribute significantly to the systematic error). A detailed breakdown of these contributions is reported in table 2

\section{Conclusions}

CDF II just begun data taking. Even with the small amount of luminosity integrated so far, our ability of performing a series of world class heavy

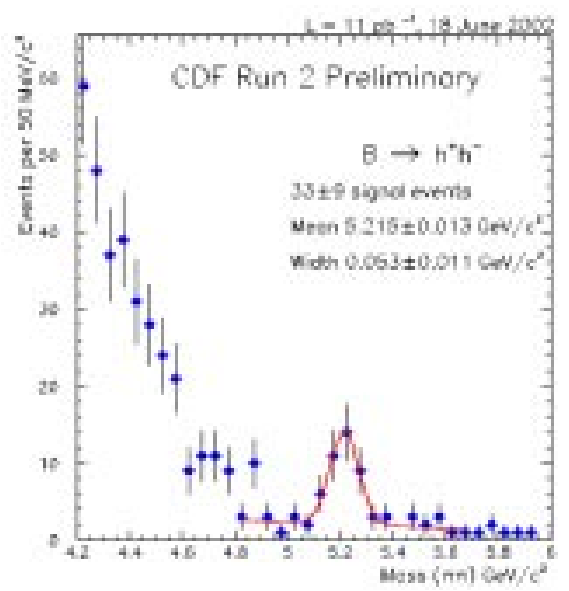

Figure 4. Invariant mass distribution for the $B \rightarrow$ $h^{+} h^{-}$decay, after appropriate selections.

flavor physics measurements is already evident. As a proof of principle, we report in figures 3 and 4 the signals $B^{ \pm} \rightarrow D^{\circ} \pi^{ \pm}$and $B \rightarrow h^{+} h^{-}$reconstructed from the hadronic SVT trigger.

As a preview of our ability, we reported in this paper the results of a preliminary measurement of $\frac{B R\left(D^{\circ} \rightarrow \pi^{+} \pi^{-}\right)}{B R\left(D^{\circ} \rightarrow K^{ \pm} \pi^{\mp}\right)}, \frac{B R\left(D^{\circ} \rightarrow K^{+} K^{-}\right)}{B R\left(D^{\circ} \rightarrow K^{ \pm} \pi^{\mp}\right)}$ and $\Delta m\left(D_{s}-D^{+}\right)$. In all cases the achieved resolution is close to the world averaged measurements and will be soon improved by better understanding of the detector and analysis systematics, together with larger statistics.

\begin{tabular}{lc}
\hline Systematic source & Syst $\left[\mathrm{MeV} / \mathrm{c}^{2}\right]$ \\
\hline$\chi^{2}$ & 0.06 \\
$L_{x y}$ & 0.09 \\
$\Theta_{\text {hel }}$ & 0.09 \\
Multiple cand. & 0.04 \\
kinematics & 0.004 \\
\hline Tracking error scale & 0.03 \\
Alignment & 0.055 \\
Material & 0.015 \\
B field & 0.025 \\
\hline fit & 0.22 \\
\hline Total & 0.273 \\
\hline
\end{tabular}

Table 2

Systematic uncertainty contribution for the measurement of the $D_{s}-D^{+}$invariant mass difference. 


\section{REFERENCES}

1. CDF II TDR, FERMILAB-Pub-96/390-E

2. B physics at the Tevatron, FERMILAB Pub01/197

3. Tevatron Measurements Related to CP violation, FERMILAB-CONF-00/272-E

4. See Ch. Paus, Heavy flavor production at $\mathrm{CDF}$, this same proceeding

5. K. Hagiwara et al., Physical Review D66, 01000001 (2002)

6. Cleo Collab., Hep-ex/0111024 